\title{
b-8. Study of Intracranial Pressure by Direct and Continuous Measurement-Second Report
}

\author{
Kinjiro Iwate, Saburo Watanabe, Hiromichi Shibahara and \\ Toshiyuki Hirota \\ Department of Surgery, Nagoya University
}

In the first report of this study, we demonstrated our newly designed intrazranial pressure measuring device consisting of a semiconductor strain gauge of $2 \mathrm{~mm}$. in thickness and $10 \mathrm{~mm}$. in diameter. With this device, the brain pressure san be measured with the accuracy of $2 \mathrm{~mm}$. of $\mathrm{H}_{2} \mathrm{O}$ and can be recorded

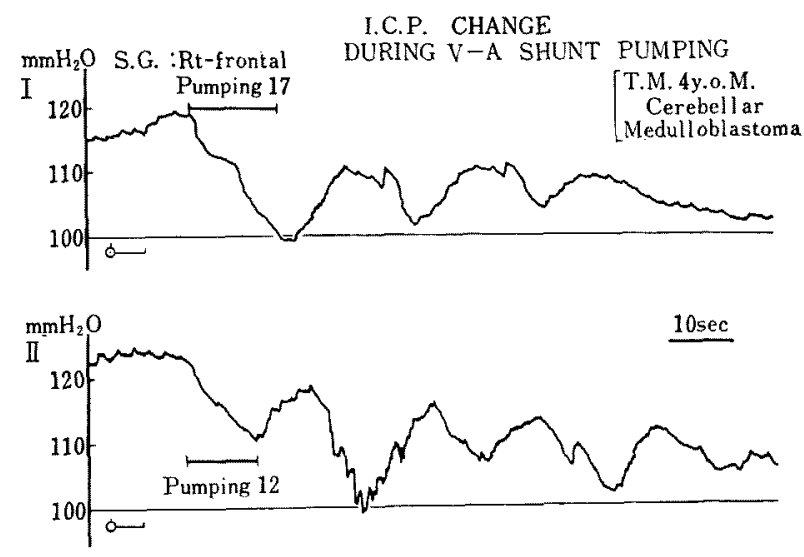

zontinuously on the recording apparatus. In addition to the pressure wave and plateaux wave of the brain pressure, a smaller pulse wave can be readily jbserved.

In the animal experiment, we confirmed that the amplitude of pulse wave increases according to the elevation of intracranial pressure. We consider this phenomenon to be the change of cushion effect of the brain parenchyma due to the elevation of brain tension; however, several other factors should be taken into account. This is interesting because observation of pulse wave may give ndirect information of increased intracranial pressure, for example, by observaion of midline echo by ultrasonic means.

In this report, we demonstrated the pattern of continuously recorded intraranial pressure in various neurosurgical procedures such as during and after raniotomies, air studies, V-A anastomic procedures or the influence of general inesthesia. The figure shown is one of the examples which shows the change of ntracranial pressure during and after the pumping of $\mathrm{V}-\mathrm{A}$ shunt. After pump$\mathrm{ng}$, the brain pressure decreases in the manner of unudlatory attenuation manner. 